\title{
Engineering of Functional Tendon
}

\author{
SARAH CALVE, B.S., ${ }^{1}$ ROBERT G. DENNIS, Ph.D., ${ }^{2-4}$ PAUL E. KOSNIK II, Ph.D., ${ }^{5}$ \\ KEITH BAAR, Ph.D., ${ }^{2,3}$ KARL GROSH, Ph.D., ${ }^{3,4}$ and ELLEN M. ARRUDA, Ph.D.1,3
}

\begin{abstract}
Surgical tendon repair is limited by the availability of viable tissue for transplantation. Because of its relatively avascular nature, tendon is a prime candidate for engineered tissue replacement. To address this problem, cells isolated from rat Achilles tendon were grown to confluence in culture and allowed to self-assemble into a cylinder between two anchor points. The resulting scaffold-free tissue was composed of aligned, small-diameter collagen fibrils, a large number of cells, and an excess of noncollagenous extracellular matrix; all characteristics of embryonic tendon. The stress-strain response of the constructs also resembles the nonlinear behavior of immature tendons, and the ultimate tensile strength is approximately equal to that of embryonic chick tendon, roughly 2 MPa. These physical and mechanical properties indicate that these constructs are the first viable tendons engineered in vitro, without the aid of artificial scaffolding.
\end{abstract}

\section{INTRODUCTION}

$\mathbf{T}$ Here are APPROXimately 33 million musculoskeletal injuries each year in the United States. The associated soft tissues, which include tendons, comprise almost $50 \%$ of these injuries. ${ }^{1}$ In some cases, the tendon is damaged beyond repair and partial or whole replacement is necessary. The ideal replacement would be autologous tendon, but transplantation is limited by the availability of viable autograft tissue and as a result clinical practice has turned to the use of synthetic materials. ${ }^{2}$ Current synthetic replacements include Dacron grafts, carbon fibers, and Silastic sheets, but these are unable to adequately restore function for the long term because of the inherent mechanical incompatibility with the in vitro environment of these materials as well as their tendency to degrade. ${ }^{3}$ A need exists for replacements that incorporate as many of the native properties of tendon as possible in order to restore function.
Tendons are densely packed connective tissues that transmit the forces between muscle and bone. They are stiff in tension yet flexible enough to conform to their anatomical environment. The material properties of tendon tissue can be attributed to the parallel fibrils of collagen that make up approximately $75 \%$ of the dry weight of adult tendons. ${ }^{4}$ In the resting state the fibrils display a periodic wavy pattern, defined as the crimp. As a tendon is stretched the crimped collagen fibrils begin to straighten out, and as a result the tendon becomes stiffer with increasing mechanical strain. Tendons have a low cell density, about $20 \%$ of the tissue volume, ${ }^{4}$ but the fibroblasts are integral in the development and maintenance of the tissue. The distinct spatial orientation of tendon fibroblasts is associated with the organization of collagen fibers into the hierarchical tendon structure. ${ }^{5}$

Because of its relatively avascular nature, tendon is a prime candidate for engineered tissue replacement. Previous attempts have been made to create biologically

\footnotetext{
${ }^{1}$ Program in Macromolecular Science and Engineering, University of Michigan, Ann Arbor, Michigan.

${ }^{2}$ Institute of Gerontology, University of Michigan, Ann Arbor, Michigan.

${ }^{3}$ Department of Mechanical Engineering, University of Michigan, Ann Arbor, Michigan.

${ }^{4}$ Department of Biomedical Engineering, University of Michigan, Ann Arbor, Michigan

${ }^{5}$ Tissue Genesis, Honolulu, Hawaii.
} 
based tendons in vitro, but these have met with limited success because of the difficulty in creating a construct that is both mechanically and biologically compatible with the in vivo environment. ${ }^{1,2,6-8}$ Mechanical difficulties can arise from the reliance on artificial scaffolds when attempting to engineer tendon. Type I collagen is one of the most widely used scaffold materials since it was observed by Bell et al. that fibroblasts will contract a collagen gel to form a tissue-like structure. ${ }^{9}$ Collagen would appear to be the ideal foundation for an artificial tendon, but at present the mechanical properties of in vitro fibroblast-collagen constructs are inferior to those of native tissues. ${ }^{10-14}$ An explanation for this discrepancy is that gelled collagen is generally disorganized and forms fibrils of physiological thickness only under stringent conditions. ${ }^{15}$ Furthermore, native tendons possess an extracellular matrix (ECM) composed of many proteins, glycosaminoglycans, and proteoglycans that control the assembly of the collagen fibril, the load-bearing unit, and contribute to the formation of the tissue hierarchy. ${ }^{16} \mathrm{Fi}-$ broblasts rely on cell-matrix signaling pathways during development to properly assemble the fibrils and maintain form and function after maturation. ${ }^{17}$ Koob and Hernandez created a mechanically relevant construct by cross-linking extruded collagen fibers with nordihydroquaiaretic acid (NDGA), a plant derived antioxidant, for which only ultimate strengths were reported and not the entire elastic response. ${ }^{8}$ Goldstein et al. used the same idea of creating a fiber composite to create artificial prostheses but relied on cross-linking methods that are cytotoxic and/or materials that are nonbiodegradable. ${ }^{2}$

To induce the formation of a tendon-like structure with adequate mechanical properties based solely on biological products, the successful engineered tendon will need to include all the necessary ECM components. The ECM will give the construct mechanical properties similar to those of the replaced tendon so that mobilization can resume as soon as possible, accelerating the process of healing. ${ }^{18,19}$ We believe that if tendon fibroblasts can be induced to secrete and assemble their own ECM, that is, secrete their own scaffold, the resulting mechanical and morphological properties will resemble those of native tendons. This approach was used to develop a method that provides appropriate environmental cues in vitro for tendon fibroblasts to generate their own ECM and assemble into a three-dimensional construct. These self-assembling tendon constructs morphologically and mechanically resemble immature tendons.

\section{MATERIALS AND METHODS}

The general method for the culture of self-organizing tissues was developed using primary skeletal muscle myogenic precursor cells. ${ }^{20}$ The following method is based in large part on the method for skeletal muscle, but has been modified in specific details to promote the selforganization of tendon tissue in vitro.

\section{Acquisition of tissue}

Primary rat tendon fibroblasts were obtained from the Achilles tendons of Fischer 344 retired breeder rats (Charles River Laboratories, Wilmington, MA). Neonatal rat Achilles tendons were harvested from 2-day-old Fischer 344 pups for transmission electron microscopy.

Achilles tendon cells were dissociated by placement in a $0.25 \%$ trypsin-EDTA solution (GIBCO-BRL, Rockville, MD) containing 100 units/mL type I collagenase (Worthington Biochemical, Lakewood, NJ). The solution was placed in a reciprocal shaking bath at $37^{\circ} \mathrm{C}$ (Precision Scientific, Winchester, VA) for $6 \mathrm{~h}$ to facilitate breakdown of the ECM. After the tissue was dissociated, the cells were pelleted by centrifugation (AccuSpin FR; Beckman Coulter, Fullerton, CA) at $100 \times g$ for $5 \mathrm{~min}$ and the supernatant was removed by aspiration. The cells were resuspended with growth medium (400 mL of Ham F-12 [GIBCO-BRL], $100 \mathrm{~mL}$ of fetal bovine serum [FBS; GIBCO-BRL] and antibiotic-antimycotic [100 units/mL; GIBCO-BRL]) and then expanded in $83-\mathrm{cm}^{2}$ tissue culture flasks (Falcon; BD Biosciences Discovery Labware, Franklin Lakes, NJ). Cells were passaged at $\sim 60 \%$ confluence and stored in liquid nitrogen until needed.

\section{Preparation of culture dishes}

An amenable substrate was created by coating 35-mm culture dishes (Falcon) with $1.5 \mathrm{~mL}$ of SYLGARD (type 184 silicone elastomer; Dow Chemical, Midland, MI) as described previously. ${ }^{20}$ After curing for 2 weeks, the dishes were rinsed with Dulbecco's phosphate-buffered saline (DPBS; GIBCO-BRL) and natural mouse laminin $\left(3.0 \mu \mathrm{g} / \mathrm{cm}^{2}\right.$; GIBCO-BRL) was applied as a $9.6-\mathrm{mg} / \mathrm{mL}$ solution of laminin in DPBS. The DPBS was allowed to evaporate overnight in a biological safety cabinet, leaving a layer of laminin-coated SYLGARD. Dishes were rinsed with DPBS and the anchors, 6-mm segments of size 0 silk suture (metric size, 3.5; Ethican, Somerville, $\mathrm{NJ})$, dipped in natural mouse laminin $(50 \mu \mathrm{g} / \mathrm{mL}$ in DPBS), were pinned $12 \mathrm{~mm}$ apart with 0.10 -mm-diameter stainless steel minutien pins (Australian Entomological Supplies, Bangalow, NSW, Australia). Sutures were allowed to dry, and the plates were filled with $1 \mathrm{~mL}$ of growth medium, enough to cover the top of the sutures. The plates were sterilized via ultraviolet irradiation (wavelength, $253.7 \mathrm{~nm}$; bulb, G30T8) in a biological safety cabinet for $90 \mathrm{~min}$ and then placed in an incubator $\left(5 \% \mathrm{CO}_{2}, 37^{\circ} \mathrm{C}\right)$ for $5-8$ days.

After incubation, the growth medium was aspirated 
and $2 \times 10^{5}$ cells suspended in $2 \mathrm{~mL}$ growth medium were seeded onto each plate and supplemented with Lascorbic acid 2-phosphate (100 $\mu \mathrm{g} / \mathrm{mL}$; Sigma-Aldrich, St. Louis, MO), a stable derivative of ascorbic acid. ${ }^{21}$ Fresh ascorbic acid was added each time the growth medium was changed, every 2-3 days. When the cells became confluent, after approximately 5 days, differentiation medium ( $465 \mathrm{~mL}$ of DMEM [GIBCO-BRL], 35 $\mathrm{mL}$ of FBS, antibiotic-antimycotic [100 units/mL]) was substituted for growth medium to induce construct formation. The differentiation medium was changed every 2-3 days until the constructs were used for testing.

\section{Histology}

Neonatal tendons and constructs were fixed, in situ, in a $3 \%$ formaldehyde-glutaraldehyde in $0.1 \mathrm{M}$ sodium cacodylate buffer solution, pH 7.4 (Electron Microscopy Sciences, Fort Washington, PA), at $4{ }^{\circ} \mathrm{C}$ and embedded in Epon (Eponate 12 resin; Ted Pella, Redding, CA). For light microscopy, semithick sections, $1 \mu \mathrm{m}$, were cut with an ultramicrotome (Sorvall, Newtown, CT) and mounted on glass microscope slides and stained with $1 \%(\mathrm{w} / \mathrm{v})$ toluidine blue solution. Ultrathin slices, $50 \mathrm{~nm}$, were cut for electron microscopy and were mounted on uncoated copper grids and stained with aqueous uranyl acetate and lead citrate. The ultrastructure of the constructs was investigated with a transmission electron microscope (Philips Medical Systems, Bothell, WA) at $60 \mathrm{kV}$.

\section{Mechanical testing of constructs}

Tendon constructs were removed from their culture dish immediately before testing. The construct diameter was measured at several positions along the length, using an inverted microscope (Axiovert 25 at $\times 50$ magnification; Carl Zeiss, Thornwood, NY). An average diameter was calculated, using all measured values. Tensile testing was performed with an 810 Material Testing System (MTS Systems, Eden Prairie, MN) outfitted with a custom optical 200-mN load cell ${ }^{22}$ and grips machined from Delrin (acetal). The grips clamped the specimens via an adjustable set screw. The tests were performed so that the grips clamped onto the construct and the suture was not subjected to a strain field. The suture material could not influence the stress-strain response because the suture was present only at the ends of the constructs and not within the gauge length. Data acquisition and control were performed with LabVIEW software (National Instruments, Austin, TX) on a Dell Precision 300 computer. The load cell was zeroed before the attachment of each sample. Samples were moistened by regularly applying drops of DPBS with a Pasteur pipet, as drying out of specimens has been shown to significantly alter the mechanical properties of tendons and other soft tissues. ${ }^{23}$ The gauge length was taken to be the length of the construct between the grips, which was measured with digital calipers after inserting and clamping the sample into both grips and applying a prestress of approximately $4 \mathrm{kPa}$. The force associated with this prestress, $1 \mathrm{mN}$, was $0.4 \%$ of the full range of the load cell, and roughly twice its resolution limit. Instead of testing at a constant displacement rate, which is the common practice, the samples were tested at a constant true strain rate of $0.05 \mathrm{~s}^{-1}$. Tendons and other soft tissues are viscoelastic, and are therefore rate dependent. To capture the true material properties the following displacement waveform was employed:

$$
\delta=l_{0}[\exp (\dot{\varepsilon} t)-1]
$$

Where $\delta$ is displacement, $l_{0}$ is the initial gauge length, $\dot{\varepsilon}$ is the true strain rate, and $t$ is time. This method ensures that the strain rate is dependent on the current length, not the initial length.

\section{RESULTS}

The process by which the cell layer self-assembles into constructs is highly repeatable. More than 50 constructs have been engineered using the protocol described above. The formation of constructs has a success rate of about $60 \%$. The remaining constructs failed to form because of improper delamination or tore soon after rolling up into a cylinder. These constructs were supported between the anchors above the SYLGARD substrate under self-mediated tension. When probed with tweezers they were noticeably taut, and when released from one anchor the constructs contracted slightly. Figure 1 demonstrates delamination of the intact cell layer and gradual self-assembly into a cylindrical construct. After the switch from growth to differentiation medium, constructs formed in about 2 weeks. No external influence was necessary for the cell layer to detach from the sides of the culture dish and SYLGARD. Variations in procedure inhibited the formation of constructs. For example, if the switch was made from growth medium to differentiation medium in four or fewer days after plating, the construct would form, but would break soon after development. The addition of ascorbic acid was also necessary to maintain the cell layer. Its absence resulted in delamination at many places within the plate instead of only at the periphery, and without an intact layer a construct could not form.

The tendon constructs display an ultrastructural morphology similar to that of neonatal rat Achilles tendon (Fig. 2). Visual inspection of the micrographs indicated that both construct and immature tendon have a relatively high cell-to-volume ratio and some disorganized ECM surrounding the collagen fibrils. The fibril diameter is $\sim 60 \mathrm{~nm}$ and displays the periodic striations characteristic of type I collagen. 


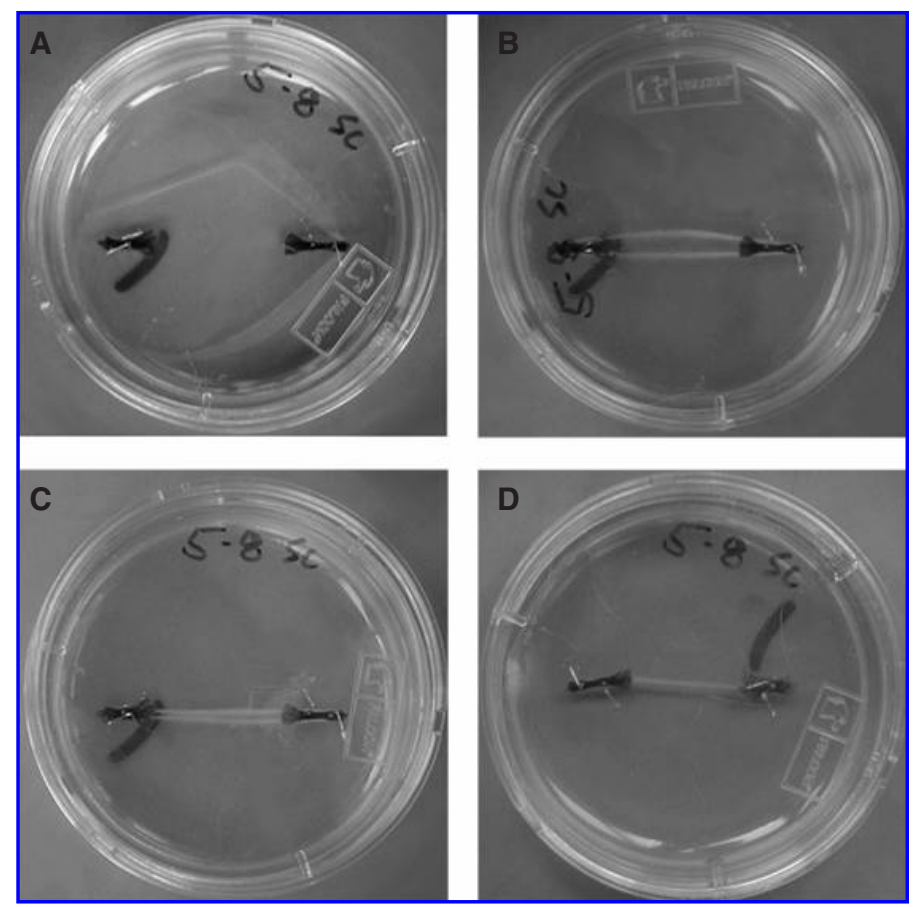

FIG. 1. Progressive delamination of a two-dimensional cell layer and formation of a tendon construct. Optimized culture variables: $5 \times 10^{5}$ cells per plate, coated with laminin $\left(2.0 \mu \mathrm{g} / \mathrm{cm}^{2}\right)$, and fed with high-serum medium for 4 days before changing to low-serum medium. Photos were taken (A) 9 days, (B) 12 days, (C) 15 days, and (D) 3 months after plating.

The stress-strain response of the tendon constructs closely resembled that of immature tendon (Fig. 3). The initial response is compliant and resembles the wellknown toe region of soft tissue response. ${ }^{24}$ At a nominal strain of 0.05 , the tangent modulus or the slope of the tangent to the stress-strain curve at 0.05 strain markedly increases, and the stress-strain response is approximately linear in the strain range of 0.11 to 0.19 , at which point

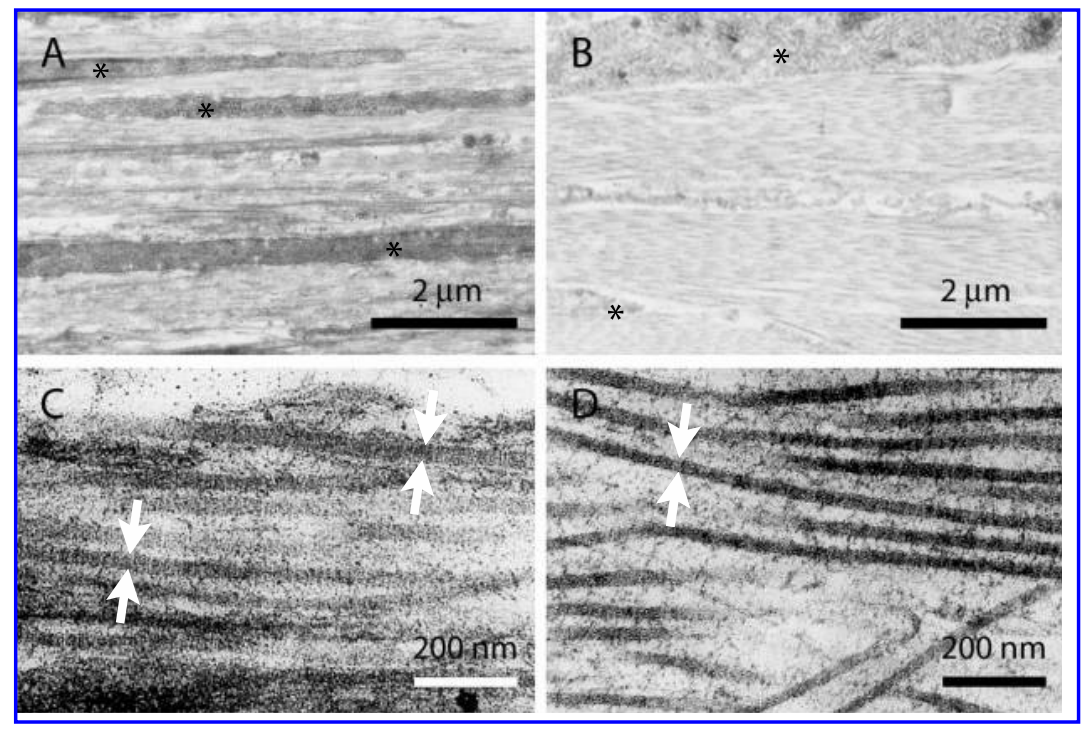

FIG. 2. Morphological comparison of a tendon construct with a 2-day-old neonatal rat tendon. (A and C) Tendon construct grown in high-serum medium for 5 days before changing to low-serum medium and seeded with $5 \times 10^{5}$ cells per plate coated with laminin at $2.0 \mu \mathrm{g} / \mathrm{cm}^{2}$. The layer started delaminating after 17 days and was fixed 18 days after the construct formed a compact cylinder. (B and D) Neonatal rat Achilles tendon fixed in situ. Note that both the tendon construct and neonatal tendon (A and B) are highly cellular (*). At higher magnification, it is evident that the fibril diameter is similar in both tissues $[\sim 50 \mathrm{~nm}$; arrowheads in (C) and (D)]. 


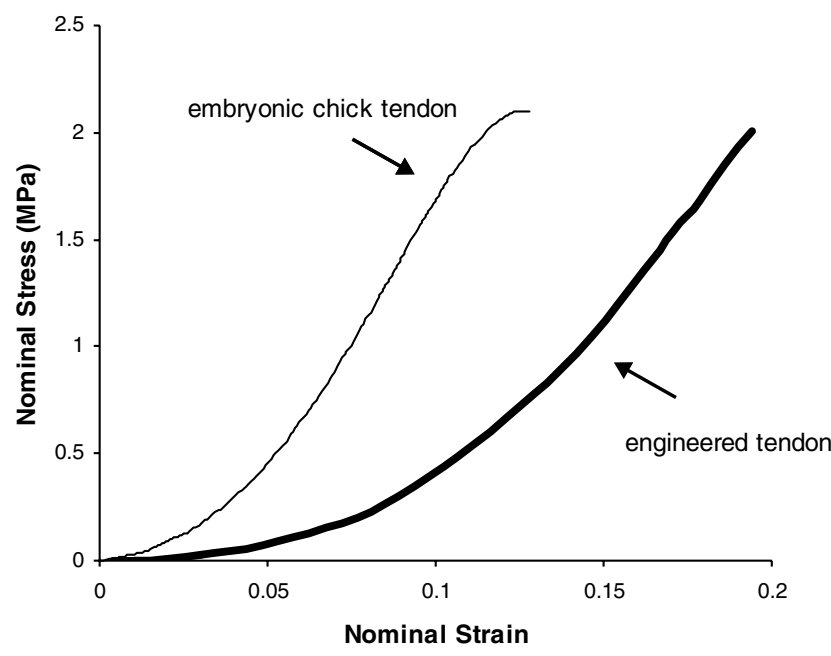

FIG. 3. Stress-strain responses of tendon construct and embryonic chicken extensor tendon (14 days in ovo). ${ }^{25}$ The tendon constructs were stressed until failure at a strain rate of 0.05 $\mathrm{s}^{-1}$. Whereas the toe region of the engineered tendon is elongated, the tangent moduli and ultimate tensile strengths of both tissue are similar.

the construct failed by breaking in one of the grips. The tangent modulus measured at a strain of 0.2 , within the linear region of the response, is $17 \mathrm{MPa}$. The constructs are mechanically similar to embryonic chicken extensor tendons, which have a tangent modulus of $27 \mathrm{MPa} .{ }^{25}$

\section{DISCUSSION}

Primary tendon fibroblasts secrete and organize their own ECM and under the right conditions will self-assemble into cylindrical constructs without the aid of exogenous scaffolding. The resulting constructs are both structurally and functionally similar to embryonic tendons.

The constructs display the nonlinear response characteristic of soft tissues, but the mechanical properties of the constructs are significantly lower than those of mature tendons. Adult rat tail tendon has a ultimate tensile strength of approximately $42 \mathrm{MPa}$ and a tangent modulus of $632 \mathrm{MPa}$, both an order of magnitude greater than the values reported for the tendon constructs. ${ }^{26}$ The large discrepancy may be attributed to the decrease in cellularity, and to the increase in both the collagen fibril diameter and organization over the course of development. ${ }^{27}$ Because the constructs are morphologically similar to neonatal tendon, the mechanical properties should resemble immature rather than mature tendon. The dearth of mechanical data on immature tendons allows for the use only of chick extensor tendons as a comparative model, and direct comparison of the chick and construct data show that they display a similar stress-strain response (Fig. 3). One difference between the tissues is the strain at failure, 0.12 for chick tendon and 0.19 for the constructs, but this may be attributed to the testing procedure rather than the intrinsic material properties of the tissues. It is unknown whether McBride et al. ${ }^{25}$ preloaded their specimens, which would decrease the length of the toe region and consequentially the strain at failure. Another cause of this discrepancy may result from the drying out of the specimens. Although both procedures entailed periodic wetting of the tissues, the degree of hydration may have been different in each of the tissues. As tendons dry out, the stiffness increases and the toe region becomes shorter. ${ }^{28}$

The integrity of the constructs was dependent on the time of medium exchange and the ascorbic acid concentration. If the medium was switched to differentiation medium before 5 days, viable constructs would not form. This is likely the result of a decrease in the production of ECM and/or the proliferation of the fibroblasts when placed in the low-serum differentiation medium, as the concentration of serum has been shown to affect both quantities. $^{29}$ The resulting constructs were not strong enough to maintain the tension generated between the sutures and prematurely broke.

In the absence of ascorbic acid, a confluent layer of fibroblasts could not be maintained. This is attributed to a decreased ability to form a stable ECM, most importantly type I collagen, which is dependent on the inclusion of ascorbic acid. ${ }^{21}$ Ishikawa et al. generated balls of fibroblasts from dermal fibroblasts grown in vitro, using a process similar to that described above, and demonstrated that fibroblasts alone were sufficient for the formation of ECM including bundles of type I collagen fibrils. ${ }^{30}$ As in the previous study, the inclusion of ascorbic acid was necessary for cell layer integrity and for proper substrate detachment. ${ }^{30}$ Whether or not the cell layers would have detached in a medium containing ascorbic acid but no serum was not investigated in either of these studies.

The constructs and immature tendons display a similar morphology (Fig. 2) ${ }^{31}$ Both exhibit a high degree of cellularity and excess ECM components, in contrast to the highly ordered mature tendon. ${ }^{5}$ These constructs have the potential to further elucidate the factors that influence tendon development. The role of specific growth factors, genes, or mechanical forces in tendon development can be studied in isolation, eliminating the confounding variables present in vivo. For example, it is generally accepted that mechanical interventions influence tendon development, but there have been few studies that explicitly address this issue. ${ }^{32-34}$ The greatest difficulty in determining the role of force on tendon development has been the isolation of the effect that force has on the tissue, independent of the influence of concurrent alterations to adjacent tissues and the overall hormonal environment. 
These constructs may provide the necessary isolated environment needed to decipher the role these factors play.

The ultimate tensile strength of the constructs may be greater than reported here because the majority of the constructs failed at either the upper or the lower grip. This is attributed to the grip pressure due to manual tightening, which could result in localized lateral compression of the construct at that interface and the introduction of a stress concentrator.

The findings herein suggest that under the right conditions, adult tendon fibroblasts rebuild tendon morphology by recapitulating the embryonic state. This return to a primitive state has also been shown in studies of tendon repair and may provide a mechanism to increase tendon plasticity. ${ }^{35}$ The work of Postacchini and De Martino on the repair of tendon after partial tenotomy demonstrated that during repair, tendon morphology progresses from developmental to mature in $\sim 16$ weeks. ${ }^{35}$ The first collagen fibrils produced have small diameters, 20-80 nm. The fibrils continue to mature and after 16 weeks the fibrils possess a diameter of $200-300 \mathrm{~nm}$. The regenerative pattern of tendon in vivo admits the hypothesis that tendon constructs have the capacity to mature in vitro when incubated in the proper environment, specifically with the application of mechanical strain.

The exact role of mechanics in the assembly and organization of tendons is currently under examination by culturing the constructs in bioreactors that will apply cyclic and static physiological loads over the course of construct development. It is hypothesized that the mechanical stimuli will induce the constructs to remodel and develop a more mature phenotype, much in the same way that embryonic movement influences the structure of developing tissues. ${ }^{32}$ These mechanical studies can also be used to clarify the roles ECM, tendon cells, and the hierarchical structure in which they are arranged play in transducing the forces from muscle to bone.

These constructs also have the potential to be used in studies of ligament function and replacement. Tendons and ligaments are generally grouped together in the literature because these tissues possess similar structural and mechanical characteristics, but there are also subtle differences that have not been thoroughly investigated. There is generally a greater and more variable amount of elastin in ligaments and the development and biochemical content of tendons and ligaments have been shown to differ. ${ }^{36,37}$ These differences may be the result of phenotypically distinct fibroblasts, loading environment, or extrinsic signals. The formation of analogous constructs, using ligament fibroblasts, may help to identify the phenotypic and mechanical differences between these tissues. Here, the constructs have been defined as tendonlike because the fibroblasts used are derived from tendons.

In summary, the current work demonstrates that func- tional immature tendons can be formed by the self-assembly of fibroblasts in vitro. These constructs are morphologically and mechanically similar to embryonic tendon and are potentially useful for studying the developmental biology of tendon as well as for clinical use in tendon repair.

\section{ACKNOWLEDGMENTS}

The authors thank Dr. Krystyna Pasyk for help in generating the images from light and transmission electron microscopy. S.C. was partially supported by a University of Michigan GE-Rackham Merit Fellowship. This work was funded in part by DARPA (Contracting Agent: SPAWAR) contract N66001-02-C-8034 and by NSF (CMS division) contract CMS 9988693.

\section{REFERENCES}

1. Butler, D.L., and Awad, H.A. Perspectives on cell and collagen composites for tendon repair. Clin. Orthop. 367, S324, 1999.

2. Goldstein, J.D., Tria, A.J., Zawadsky, J.P., et al. Development of a reconstituted collagen tendon prosthesis: A preliminary implantation study. J. Bone Joint Surg. Am. 71, 1183, 1989.

3. Iannace, S., Sabatini, G., Ambrosio, L., et al. Mechanicalbehavior of composite artificial tendons and ligaments. Biomaterials 16, 675, 1995.

4. Nordin, M., Lorenz, T., and Campello, M. Biomechanics of tendons and ligaments. In: Nordin, M., and Frankel, V.H., eds. Basic Biomechanics of the Musculoskeletal System. New York: Lippincott Williams \& Wilkins, 2001, pp. 102-125.

5. McBride, D.J., Hahn, R.A., and Silver, F.H. Morphological characterization of tendon development during chick embryogenesis: Measurement of birefringence retardation. Int. J. Biol. Macromol. 7, 71, 1985.

6. Torres, D.S., Freyman, T.M., Yannas, I.V., et al. Tendon cell contraction of collagen-GAG matrices in vitro: Effect of cross-linking. Biomaterials 21, 1607, 2000.

7. Cao, Y.L., Liu, Y.T., Liu, W., et al. Bridging tendon defects using autologous tenocyte engineered tendon in a hen model. Plast. Reconstr. Surg. 110, 1280, 2002.

8. Koob, T.J., and Hernandez, D.J. Material properties of polymerized NDGA-collagen composite fibers: Development of biologically based tendon constructs. Biomaterials 23, 203, 2002.

9. Bell, E., Ivarsson, B., and Merrill, C. Production of a tissue-like structure by contraction of collagen lattices by human-fibroblasts of different proliferative potential in vitro. Proc. Natl. Acad. Sci. U.S.A. 76, 1274, 1979.

10. Huang, D., Chang, T.R., Aggarwal, A., et al. Mechanisms and dynamics of mechanical strengthening in ligamentequivalent fibroblast-populated collagen matrices. Ann. Biomed. Eng. 21, 289, 1993. 
11. Wakatsuki, T., Kolodney, M.S., Zahalak, G.I., et al. Cell mechanics studied by a reconstituted model tissue. Biophys. J. 79, 2353, 2000.

12. Seliktar, D., Black, R.A., Vito, R.P., et al. Dynamic mechanical conditioning of collagen-gel blood vessel constructs induces remodeling in vitro. Ann. Biomed. Eng. 28, 351, 2000.

13. Brown, R.A., Prajapati, R., McGrouther, D.A., et al. Tensional homeostasis in dermal fibroblasts: Mechanical responses to mechanical loading in three-dimensional substrates. J. Cell. Physiol. 175, 323, 1998.

14. Cacou, C., Palmer, D., Lee, D.A., et al. A system for monitoring the response of uniaxial strain on cell seeded collagen gels. Med. Eng. Phys. 22, 327, 2000.

15. Holmes, D.F., Capaldi, M.J., and Chapman, J.A. Reconstitution of collagen fibrils in vitro: The assembly process depends on the initiating procedure. Int. J. Biol. Macromol. 8, 161, 1986.

16. Birk, D.E., and Zycband, E. Assembly of the tendon extracellular matrix during development. J. Anat. 184, 457, 1994.

17. Nurminskaya, M.V., and Birk, D.E. Differential expression of genes associated with collagen fibril growth in the chicken tendon: Identification of structural and regulatory genes by subtractive hybridization. Arch. Biochem. Biophys. 350, 1, 1998.

18. Murrell, G.A.C., Lilly, E.G., Goldner, R.D., et al. Effects of immobilization on achilles-tendon healing in a rat model. J. Orthop. Res. 12, 582, 1994.

19. Gelberman, R.H., Vandeberg, J.S., Manske, P.R., et al. The early stages of flexor tendon healing: A morphologic study of the 1st 14 days. J. Hand Surg. Am. 10, 776, 1985.

20. Dennis, R.G., and Kosnik, P.E. Excitability and isometric contractile properties of mammalian skeletal muscle constructs engineered in vitro. In Vitro Cell. Dev. Biol. Anim. 36, 327, 2000.

21. Kurata, S., and Hata, R. Epidermal growth-factor inhibits transcription of type-I collagen genes and production of type-I collagen in cultured human skin fibroblasts in the presence and absence of L-ascorbic acid 2-phosphate, a long-acting vitamin-C derivative. J. Biol. Chem. 266, 9997, 1991.

22. Dennis, R.G., and Kosnik, P.E. Mesenchymal cell culture: Instrumentation and methods for evaluating engineered muscle. In: Atala, A., and Lanza, R.P., eds. Methods of Tissue Engineering. New York: Academic Press, 2002, pp. 307-315.

23. Betsch, D.F., and Baer, E. Structure and mechanical-properties of rat tail tendon. Biorheology 17, 83, 1980.

24. Fung, Y.C. Biomechanics: Mechanical Properties of Living Tissues, 2nd Ed. New York: Springer-Verlag, 1993.

25. McBride, D.J., Trelstad, R.L., and Silver, F.H. Structural and mechanical assessment of developing chick tendon. Int. J. Biol. Macromol. 10, 194, 1988.

26. Cartmell, J.S., and Dunn, M.G. Effect of chemical treatments on tendon cellularity and mechanical properties. J. Biomed. Mater. Res. 49, 134, 2000.

27. Moore, M.J., and Debeaux, A. A quantitative ultrastructural-study of rat tendon from birth to maturity. J. Anat. 153, 163, 1987.

28. Haut, T.L., and Haut, R.C. The state of tissue hydration determines the strain-rate-sensitive stiffness of human patellar tendon. J. Biomech. 30, 79, 1997.

29. Kang, H.J., and Kang, E.S. Ideal concentration of growth factors in rabbit's flexor tendon culture. Yonsei Med. J. 40, 26, 1999.

30. Ishikawa, O., Kondo, A., Okada, K., et al. Morphological and biochemical analyses on fibroblasts and self-produced collagens in a novel three-dimensional culture. Br. J. Dermatol. 136, 6, 1997.

31. Parry, D.A.D., and Craig, A.S. Growth and development of collagen fibrils in connective tissue. In: Ruggeri, A., and Motta, P.M., eds. Ultrastructure of the Connective Tissue Matrix. Boston: M. Nijhoff, 1984, pp. 34-64.

32. Hall, B.K., and Herring, S.W. Paralysis and growth of the musculoskeletal system in the embryonic chick. $\underline{\text { J. Mor- }}$ phol. 206, 45, 1990.

33. Germiller, J.A., Lerner, A.L., Pacifico, R.J., et al. Muscle and tendon size relationships in a paralyzed chick embryo model of clubfoot. J. Pediatr. Orthop. 18, 314, 1998.

34. Beckham, C., Dimond, R., and Greenlee, T.K. Role of movement in development of a digital flexor tendon. Am. J. Anat. 150, 443, 1977.

35. Postacchini, F., and De Martino, C. Regeneration of rabbit calcaneal tendon maturation of collagen and elastic fibers following partial tenotomy. Connect. Tissue Res. 8, 41, 1980.

36. Watanabe, M., Nojima, M., Shibata, T., et al. Maturationrelated biochemical-changes in swine anterior cruciate ligament and tibialis posterior tendon. J. Orthop. Res. 12, 672, 1994.

37. Amiel, D., Frank, C., Harwood, F.L., et al. Tendons and ligaments: A morphological and biochemical comparison. J. Orthop. Res. 1, 257, 1984.

Address reprint requests to: Ellen M. Arruda, Ph.D. Department of Mechanical Engineering University of Michigan 2250 G.G. Brown Ann Arbor, MI 48109-2125

E-mail: arruda@umich.edu 


\section{This article has been cited by:}

1. Hiromichi Omae, Chunfeng Zhao, Yu Long Sun, Kai-Nan An, Peter C. Amadio. 2009. Multilayer tendon slices seeded with bone marrow stromal cells: A novel composite for tendon engineering. Journal of Orthopaedic Research 27:7, 937-942. [CrossRef]

2. H. Narayanan, E. M. Arruda, K. Grosh, K. Garikipati. 2009. The micromechanics of fluid-solid interactions during growth in porous soft biological tissue. Biomechanics and Modeling in Mechanobiology 8:3, 167-181. [CrossRef]

3. Antonio Gigante, Eugenio Cesari, Alberto Busilacchi, Sandra Manzotti, Kyriaki Kyriakidou, Francesco Greco, Roberto Di Primio, Monica Mattioli-Belmonte. 2009. Collagen I membranes for tendon repair: Effect of collagen fiber orientation on cell behavior. Journal of Orthopaedic Research 27:6, 826-832. [CrossRef]

4. Jennifer Z. Paxton , Kenneth Donnelly , Robert P. Keatch , Keith Baar . 2009. Engineering the Bone-Ligament Interface Using Polyethylene Glycol Diacrylate Incorporated with HydroxyapatiteEngineering the Bone-Ligament Interface Using Polyethylene Glycol Diacrylate Incorporated with Hydroxyapatite. Tissue Engineering Part A 15:6, 1201-1209. [Abstract] [PDF] [PDF Plus]

5. Angela M. Throm, Wai-Ching Liu, Chi-Hung Lock, Kristen L. Billiar. 2009. Development of a cell-derived matrix: Effects of epidermal growth factor in chemically defined culture. Journal of Biomedical Materials Research Part A 9999A, NA-NA. [CrossRef]

6. James W. S. Hayami, Denver C. Surrao, Stephen D. Waldman, Brian G. Amsden. 2009. Design and characterization of a biodegradable composite scaffold for ligament tissue engineering. Journal of Biomedical Materials Research Part $A$ 9999A, NA-NA. [CrossRef]

7. Fatima N. Syed-Picard , Lisa M. Larkin , Charles M. Shaw , Ellen M. Arruda . 2009. Three-Dimensional Engineered Bone from Bone Marrow Stromal Cells and Their Autogenous Extracellular MatrixThree-Dimensional Engineered Bone from Bone Marrow Stromal Cells and Their Autogenous Extracellular Matrix. Tissue Engineering Part A 15:1, 187-195. [Abstract] [PDF] [PDF Plus]

8. Floor van Eijk, Daniel B.F. Saris, Laura B. Creemers, Jens Riesle, W. Jaap Willems , Clemens A. van Blitterswijk, Abraham J. Verbout, Wouter J.A. Dhert . 2008. The Effect of Timing of Mechanical Stimulation on Proliferation and Differentiation of Goat Bone Marrow Stem Cells Cultured on Braided PLGA ScaffoldsThe Effect of Timing of Mechanical Stimulation on Proliferation and Differentiation of Goat Bone Marrow Stem Cells Cultured on Braided PLGA Scaffolds. Tissue Engineering Part A 14:8, 1425-1433. [Abstract] [PDF] [PDF Plus]

9. Dima Sheyn, Gadi Pelled, Yoram Zilberman, Farahnaz Talasazan, Jonathan M. Frank, Dan Gazit, Zulma Gazit. 2008. Nonvirally Engineered Porcine Adipose Tissue-Derived Stem Cells: Use in Posterior Spinal Fusion. Stem Cells 26:4, 1056-1064. [CrossRef]

10. A. Menzel. 2007. A fibre reorientation model for orthotropic multiplicative growth. Biomechanics and Modeling in Mechanobiology 6:5, 303-320. [CrossRef]

11. R.K. Birla , Y.C. Huang , R.G. Dennis . 2007. Development of a Novel Bioreactor for the Mechanical Loading of Tissue-Engineered Heart MuscleDevelopment of a Novel Bioreactor for the Mechanical Loading of Tissue-Engineered Heart Muscle. Tissue Engineering 13:9, 2239-2248. [Abstract] [PDF] [PDF Plus]

12. Michelle Hairfield-Stein, Christopher England, Hyun J. Paek, Kerry B. Gilbraith, Robert Dennis, Eugene Boland , Paul Kosnik . 2007. Development of Self-Assembled, Tissue-Engineered Ligament from Bone Marrow Stromal CellsDevelopment of Self-Assembled, Tissue-Engineered Ligament from Bone Marrow Stromal Cells. Tissue Engineering 13:4, 703-710. [Abstract] [PDF] [PDF Plus]

13. Michelle Hairfield-Stein, Christopher England, Hyun J. Paek, Kerry B. Gilbraith, Robert Dennis, Eugene Boland, Paul Kosnik. 2007. Development of Self-Assembled, Tissue-Engineered Ligament from Bone Marrow Stromal Cells. Tissue Engineering, ahead of print070116053433001. [CrossRef] 
14. Michelle Hairfield-Stein, Christopher England, Hyun J. Paek, Kerry B. Gilbraith, Robert Dennis, Eugene Boland, Paul Kosnik. 2007. Development of Self-Assembled, Tissue-Engineered Ligament from Bone Marrow Stromal Cells. Tissue Engineering, ahead of print070108073551001. [CrossRef]

15. P.O. Bagnaninchi, Y. Yang, N. Zghoul, N. Maffulli, R.K. Wang, A.J. El Haj. 2007. Chitosan Micro-Channel Scaffolds for Tendon Tissue Engineering Characterized Using Optical Coherence Tomography. Tissue Engineering, ahead of print070104053042001. [CrossRef]

16. Caroline Androjna , Rebecca K. Spragg, Kathleen A. Derwin . 2007. Mechanical Conditioning of Cell-Seeded Small Intestine Submucosa: A Potential Tissue-Engineering Strategy for Tendon Repair. Tissue Engineering 13:2, 233-243. [Abstract] [PDF] [PDF Plus]

17. P.O. Bagnaninchi, Y. Yang, N. Zghoul, N. Maffulli , R.K. Wang, A.J. El Haj . 2007. Chitosan Microchannel Scaffolds for Tendon Tissue Engineering Characterized Using Optical Coherence Tomography. Tissue Engineering 13:2, 323-331. [Abstract] [PDF] [PDF Plus]

18. Milena Fini, Paola Torricelli, Gianluca Giavaresi, Roberto Rotini, Alessandro Castagna, Roberto Giardino. 2007. In vitro study comparing two collageneous membranes in view of their clinical application for rotator cuff tendon regeneration. Journal of Orthopaedic Research 25:1, 98-107. [CrossRef]

19. Caroline Androjna, Rebecca K. Spragg, Kathleen A. Derwin. 2007. Mechanical Conditioning of Cell-Seeded Small Intestine Submucosa: A Potential Tissue-Engineering Strategy for Tendon Repair. Tissue Engineering, ahead of print070101195214001. [CrossRef]

20. Zhonggang Feng, Yu Tateishi, Yasutomo Nomura, Tatsuo Kitajima, Takao Nakamura. 2007. Construction of fibroblast-collagen gels with orientated fibrils induced by static or dynamic stress: toward the fabrication of small tendon grafts. Journal of Artificial Organs 9:4, 220-225. [CrossRef]

21. Thomas Weigel, Gregor Schinkel, Andreas Lendlein. 2006. Design and preparation of polymeric scaffolds for tissue engineering. Expert Review of Medical Devices 3:6, 835-851. [CrossRef]

22. Lisa M. Larkin, Sarah Calve, Tatiana Y. Kostrominova, Ellen M. Arruda. 2006. Structure and Functional Evaluation of Tendon?Skeletal Muscle Constructs Engineered in Vitro. Tissue Engineering, ahead of print061012064037003. [CrossRef]

23. Lisa M. Larkin , Sarah Calve, Tatiana Y. Kostrominova , Ellen M. Arruda . 2006. Structure and Functional Evaluation of Tendon-Skeletal Muscle Constructs Engineered in VitroStructure and Functional Evaluation of Tendon-Skeletal Muscle Constructs Engineered in Vitro. Tissue Engineering 12:11, 3149-3158. [Abstract] [PDF] [PDF Plus]

24. Melinda Aliza Costa , Cindy Wu , Bryant Vu Pham , Alphonsus Khin Sze Chong , Hung Minh Pham , James Chang . 2006. Tissue Engineering of Flexor Tendons: Optimization of Tenocyte Proliferation Using Growth Factor SupplementationTissue Engineering of Flexor Tendons: Optimization of Tenocyte Proliferation Using Growth Factor Supplementation. Tissue Engineering 12:7, 1937-1943. [Abstract] [PDF] [PDF Plus]

25. Melinda Aliza Costa, Cindy Wu, Bryant Vu Pham, Alphonsus Khin Sze Chong, Hung Minh Pham, James Chang. 2006. Tissue Engineering of Flexor Tendons: Optimization of Tenocyte Proliferation Using Growth Factor Supplementation. Tissue Engineering, ahead of print060802052515016. [CrossRef]

26. Dejun Cao, Wei Liu, Xian Wei, Feng Xu, Lei Cui, Yilin Cao. 2006. In vitro Tendon Engineering with Avian Tenocytes and Polyglycolic Acids: A Preliminary Report. Tissue Engineering, ahead of print060517070412001. [CrossRef]

27. Dejun Cao, Wei Liu , Xian Wei , Feng Xu , Lei Cui , Yilin Cao . 2006. In Vitro Tendon Engineering with Avian Tenocytes and Polyglycolic Acids: A Preliminary ReportIn Vitro Tendon Engineering with Avian Tenocytes and Polyglycolic Acids: A Preliminary Report. Tissue Engineering 12:5, 1369-1377. [Abstract] [PDF] [PDF Plus] 
28. R. A. Brown, M. Wiseman, C.-B. Chuo, U. Cheema, S. N. Nazhat. 2005. Ultrarapid Engineering of Biomimetic Materials and Tissues: Fabrication of Nano- and Microstructures by Plastic Compression. Advanced Functional Materials 15:11, 1762-1770. [CrossRef] 